\title{
The New Great Imitator
}

\author{
Esther Nivasch ${ }^{1 *}$ and Ifeanyichukwu Anidi ${ }^{2}$ \\ ${ }^{1}$ Perelman School of Medicine, Philadelphia, United States \\ ${ }^{2}$ Hospital of the University of Pennsylvania, United States
}

\begin{abstract}
Sarcoidosis is a disease of involving the unusual accumulation on inflammatory cells which forms lumps called as granulomas. Though the pathogenesis remains undefined, it has been proposed that an initial CD4+ T cell infiltration is the spark in the inflammatory cascade that results in granulomatous formation in affected organs. In this case report we have described an unusual presentation of sarcoidosis detailing the mutable iterations of this disease.
\end{abstract}

Keywords: Sarcoidosis; Anaemia; Granulomas

\section{Introduction}

Sarcoidosis is a multi-system granulomatous disease of unknown etiology characterized by the accumulation of non-caseating granulomas, T lymphocytes, and phagocytes in diseased tissue affecting 10/100,000 persons each year [1]. Though the pathogenesis remains undefined, it has been proposed that an initial CD4+ T cell infiltration is the spark in the inflammatory cascade that results in granulomatous formation in affected organs [2]. Sarcoidosis' granulomatous infiltrate may resolve without sequelae or progress to fibrosis of the affected tissues, often manifesting as restrictive pulmonary symptoms in the $90 \%$ of cases with lung involvement [3]. Common presenting symptoms include cough, dyspnea and chest pain, sometimes accompanied by fatigue and weight loss [4]. It can also have extra-pulmonary manifestations in up to $30 \%$ of cases, affecting the skin, eyes, reticuloendothelial system, musculoskeletal system, exocrine glands, heart, kidney, and central nervous system [5]. Several classic presentations can be diagnosed as sarcoidosis without a biopsy. These include Löfgren syndrome (erythema nodosum, hilar adenopathy, migratory polyarthralgia and fever), Heerfordt's syndrome (uveoparotid fever), and asymptomatic bilateral hilar adenopathy. In all other presentations, sarcoidosis is a diagnosis of exclusion, and is established when clinico-radiographic findings - most often bilateral hilar lymphadenopathy - are supported by histologic evidence of non-caseating granulomatous inflammation [6]. Given sarcoidosis clinical variability, it is possible that it is underdiagnosed in atypical presentations.

\section{Case Report}

A 52-year-old man was evaluated at an outside hospital for bilateral lower extremity weakness, cachexia and anaemia. Over the past 18 months, he had experienced a $41 \mathrm{~kg}(90 \mathrm{lb})$ unintentional weight loss without anorexia, as well as 6 months of progressive left greater than right lower extremity weakness resulting in near-paralysis such that he required a wheelchair for transport. He also reported numbness and paresthesias in his lower extremities for several months. His medical history was notable for hypertension and type 2 diabetes mellitus. The patient initially presented to an outside hematologist previous several months and was noted to have neutropenia and thrombocytopenia. Chest, abdomen and pelvis computed tomography (CT) scans were performed for suspected malignancy and demonstrated splenomegaly with hypodense lesions in the spleen as well as bronchiolitis of the lungs and reactive lymph nodes. Lumbar spine CT was obtained to assess for neurologic findings, which showed spinal stenosis and degenerative joint disease. Bone marrow biopsy (with cytogenetics), serum electropheresis, HIV serology, and hepatitis B and C serologies were performed at an outside hospital 3 months prior to our evaluation and were reportedly unremarkable apart from mildly hypocellular marrow. The patient was subsequently referred to our institution's hematology clinic for further evaluation. He reported that more than $50 \%$ of waking hours were spent in wheelchair and denied infectious symptoms On examination, he was afebrile and noted to have profound lower extremity weakness, splenomegaly and cachexia with temporal wasting; no adenopathy was appreciated. Given his concerning presentation, he was admitted to our hospital for expedited workup. Admission labs demonstrated persistent leukocytopenia, neutropenia and thrombocytopenia with WBC, neutrophil count and platelet levels at 1,600 cells per cubic millimeter, 930 cells per cubic millimeter, and 100,000 cells per cubic millimeter respectively. Imaging including chest and abdominal CTs were obtained to compare with previous scans. Chest CT was notable for centrilobular nodules and tree-inbud opacities consistent with bronchiolitis infectious or inflammatory in origin. Abdominal CT demonstrated splenomegaly with diffuse hypodense nodules noted on previous imaging, subtle lucencies in the L2 and L4 spinous processes, and a mixed lucent and sclerotic lesion in the posterior left ilium of unclear etiology. Cervical spine MRI did not reveal any evidence of cord compression. EMG studies demonstrated severe demyelinating polyneuropathy. Further workup included serum free light chain testing, which was notable for elevated levels of kappa light chains at $38.8 \mathrm{mg} / \mathrm{L}$ and a mildly elevated $\mathrm{K}: \mathrm{L}$ ratio of 1.788 . Given these findings and his significant polyneuropathy, our differential included POEMS (Polyneuropathy, Organomegaly, Endocrinopathy, Monoclonal protein, Skin changes) syndrome, multiple myeloma or an undifferentiated myelodysplastic syndrome. Elevated serum or plasma vascular endothelial growth factor (VEGF) levels are a strong indication of POEMS syndrome. However, further workup for a blood dyscrasia (including vascular endothelial growth factor levels, quantitative immunoglobulin levels, serum protein electrophoresis, urine electrophoresis, and 24-hour urine protein levels were unremarkable. A bronchoscopy was subsequently performed given the patient's radiographic evidence of pulmonary nodules; pathology of a left hilar lymph node sample revealed "bronchial mucosa with chronic non-necrotizing granulomatous inflammation and associated multinucleated giant cells," consistent with a diagnosis

*Corresponding author: Esther Nivasch, University of Pennsylvania Perelman School of Medicine, 3400 Civic Center Boulevard, Philadelphia, United States, Tel: 9143297064; E-mail: esther.nivasch@uphs.upenn.edu

Received September 02, 2017; Accepted September 15, 2017; Published September 19, 2017

Citation: Nivasch E, Anidi I (2017) The New Great Imitator. J Pulm Respir Med 7 423. doi: $10.4172 / 2161-105 X .1000423$

Copyright: (๑) 2017 Nivasch E, et al. This is an open-access article distributed unde the terms of the Creative Commons Attribution License, which permits unrestricted use, distribution, and reproduction in any medium, provided the original author and source are credited. 
of sarcoidosis. He was subsequently started on an oral glucocorticoid regimen of prednisone $50 \mathrm{mg}$ daily and discharged with establishment of outpatient neurology and pulmonology care.

\section{Diagnostic Methods}

\section{CT Chest w/IV contrast}

CT of the chest was performed from the lung apices to the lung bases, following intravenous administration of $100 \mathrm{ml}$ of Isovue- 370 contrast. Images were obtained at inspiration and viewed in axial (including thin sections), sagittal and coronal planes.

\section{CT Abdomen/Pelvis w/IV contrast}

CT examination of the abdomen and pelvis was performed from the domes of the diaphragms to the symphysis pubis after administration of oral and $100 \mathrm{ml}$ of Isovue 370 contrast.

\section{Electromyography (EMG)}

EMG was performed during which muscle activity at needle insertion and spontaneous activity at complete rest was measured. Motor unit action potential (MUAP) qualitative and quantitative waveform analyses in addition to recruitment patterns were assessed.

\section{MRI cervical spine w/o IV contrast}

Routine unenhanced MRI of the cervical spine. Sagittal STIR imaging was repeated second time due to motion degradation.

\section{Bronchoscopy}

$10 \mathrm{cc} 1 \%$ lidocaine given topically to airways during case. The bronchoscope was passed with ease through the nares; it was extended to the pharynx, larynx, trachea, right bronchial tree, and left bronchial tree. The views were excellent. The patient's toleration of the procedure was excellent.

\section{Transbronchial biopsy}

The case is received in 2 formalin-filled containers, each labeled with the patient's name and medical record number. Specimen \#1 is designated as "Transbronchial Biopsy LUL" and consists of 4 tan, irregular soft tissue fragments measuring $0.5 \mathrm{~cm}$ in greatest dimension. The specimen is submitted in toto in cassette $1 \mathrm{~A}$. Specimen $\# 2$ is designated as "Endobronchial Biopsy L Hilum " and consists of 2 tan, irregular soft tissue fragments measuring $0.6 \mathrm{~cm}$ in greatest dimension. The specimen is submitted in toto in cassette $2 \mathrm{~A}$.

\section{Results}

\section{CT Chest w/IV contrast}

Chest CT showed extensive pulmonary nodules and no destructive lytic osseous lesions in the thorax which would have suggested a diagnosis of multiple myeloma. It also excluded bronchiolitis as a likely etiology for the pulmonary nodules, given a lack of centrilobular distribution. The read also noted a constellation of "innumerable diminutive perilymphatic pulmonary nodules, mild mediastinal and bilateral hilar lymphadenopathy and moderate splenomegaly with numerous hypodense/hypoenhancing nodules," suggestive of sarcoidosis (Figure 1).

\section{CT Abdomen/Pelvis w/IV contrast}

Scan showed Splenomegaly with diffuse hypodense nodules throughout the spleen, subtle lucencies in the L2 and L4 spinous processes, and mixed lucent and sclerotic lesion in theposterior left ilium, and abnormal asymmetric sclerosis of the left femoral head. The read suggested Multiple Myeloma as a potential cause of these abnormalities (Figure 2).

\section{Electromyography (EMG)}

The EMG was significant for severe neuropathy with axonal degeneration and features of demyelination. Several etiologies can explain these findings, including toxins, rheumatological diseases and hormone abnormalities such as hypothyroidism, making the EMG findings insufficient to narrow down a diagnosis.

\section{MRI cervical spine w/o IV contrast}

The cervical MRI demonstrated multilevel spondylotic changes in the cervical spine without significant narrowing or stenosis, making spinal compression as an etiology of his symptoms unlikely.

\section{Transbronchial biopsy}

L hilar biopsy showed bronchial mucosa with chronic nonnecrotizing granulomatous inflammation and associated multinucleated giant cells, establishing the diagnosis of sarcoidosis (Figure 3).
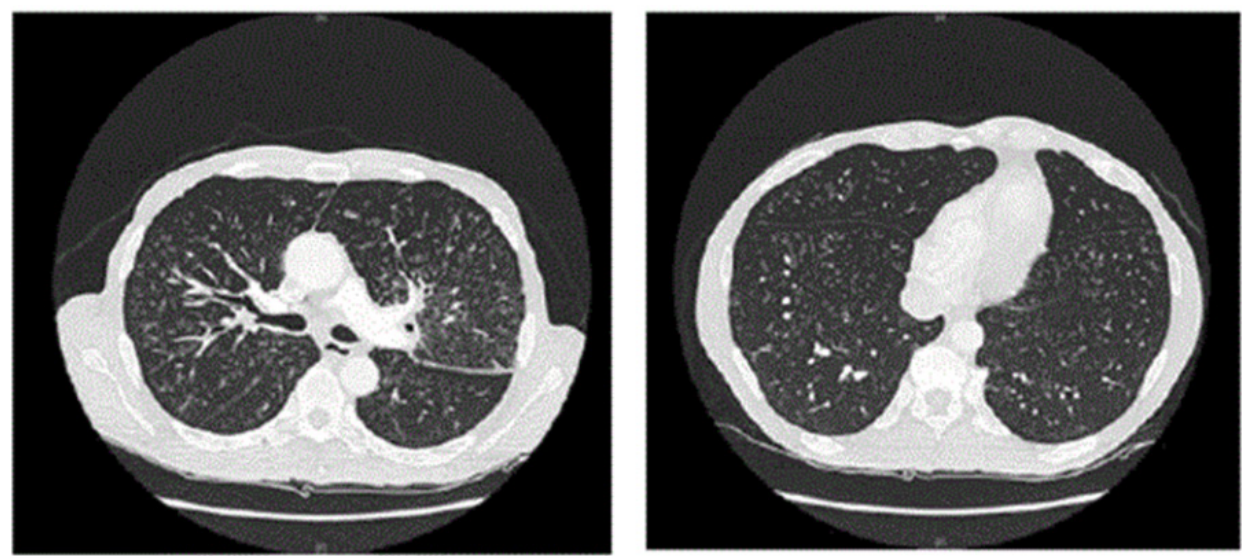

Figure 1: Chest CT with IV contrast performed during hospitalisation showed bilateral upper lobe predominant nodules and tree in bud and mils mediastinal and bilateral hilar lymphadenopathy. Evaluation of the lung parenchyma on thin section high-resolution images reveal constellation of innumerable diminutive perilymphatic pulmonary nodules, mild mediastinal and bilateral hilar lymphadenopathy and moderate splenomegaly with numerous hypodense/hypoenhancing nodules. 

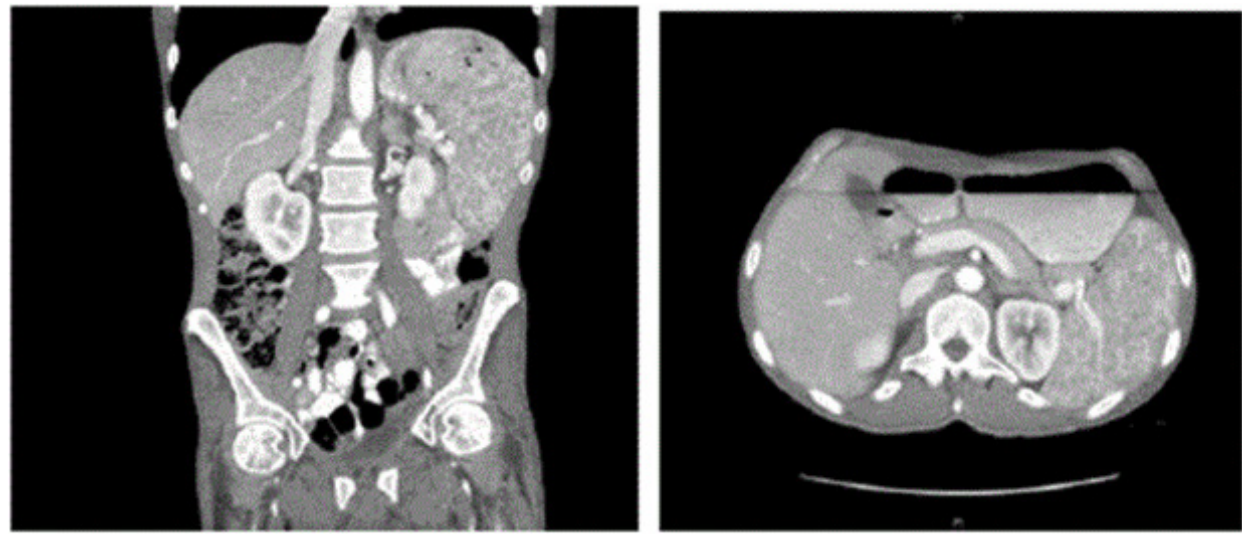

Figure 2: AP abdominal CT films with IV contrast demonstrated splenomegaly with diffuse hypodense nodules throughout the spleen.
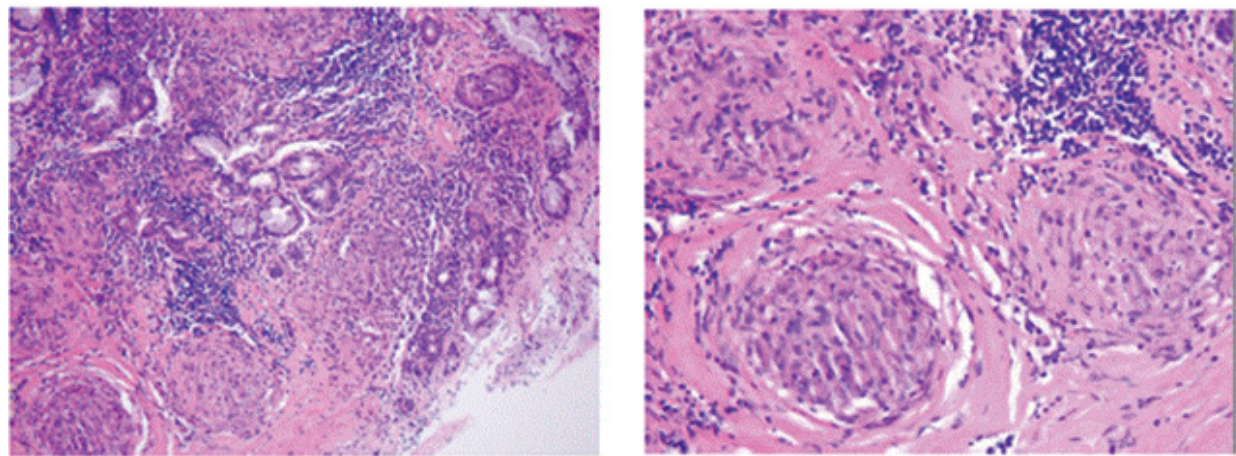

Figure 3: H\&E Staining of transbronchial biopsies demonstrating non-caseating granulomas and lymphocytic infiltration.

\section{Discussion}

Sarcoidosis is a systemic disease characterized by granulomatous inflammation. While sarcoidosis may involve several organ systems, pulmonary manifestations are typical, with $85-95 \%$ of cases affecting the mediastinopulmonary sphere [7]. Pulmonary involvement ranges from asymptomatic lymphadenopathy to chronic interstitial fibrosis refractory to medical treatment, with persistent cough being the most common presenting pulmonary symptom [8]. Given the variety of potential presentations for pulmonary sarcoidosis, clinicians frequently rely on chest X-ray imaging for diagnosis. Bilateral hilar adenopathy increases suspicion in an otherwise asymptomatic patient and may be considered diagnostic in a Löfgren-type presentation with bilateral hilar adenopathy, erythema nodosum and arthritis [9]. CT imaging is often a more helpful component of the workup for sarcoidosis. Typical CT findings include bilateral hilar lymphadenopathy, a reticulonodular pattern, perilymphatic nodule distribution, and predominant upper and middle zone parenchymal abnormalities [10].

Our case presents an interesting intersection between common and uncommon sarcoidosis presentations. Our patient's pulmonary presentation alone is not unusual for sarcoidosis: lack of symptoms, radiographic evidence of bilateral hilar lymphadenopathy and perilymphatic pulmonary nodules, and non-caseating granulomas on lymph node biopsy. However, our patient's severe and extensive extra-pulmonary symptoms including cachexia, neurodegeneration, and fatigue were very unusual for sarcoidosis and initially suggested malignancy as a more probable etiology. Sarcoidosis may have extrapulmonary symptoms in up to $30 \%$ of cases. Cutaneous, ocular, lymphatic, and hepatic involvement are most common, presenting in $11-15 \%$ of patients [11] though sarcoidosis' extra-pulmonary manifestations are many and varied. Cutaneous lesions differ and may include papular, plaque-like, nodular, and subcutaneous manifestations or lupus pernio in addition to the erythema nodosum findings seen in Löfgren's syndrome [12]. Uveitis, keratoconjunctivitis sicca, and eyelid/conjunctival granulomas are common ocular manifestations and are in fact the presenting symptoms in $5 \%$ of sarcoid patients [3]. The degree of sarcoid cardiac involvement remains unclear but may result in life-threatening complications including heart block, arrhythmias, valvular dysfunction and heart failure. Musculoskeletal involvement may include polyarthritis as noted in Löfgren's syndrome [13]. The splenic and neurologic complications of sarcoidosis seen in our patient are typically seen in no more than $8 \%$ of patients. Spleen size may span up to $18 \mathrm{~cm}$ in severe cases of sarcoidosis, and degree of splenomegaly may correlate with other markers of disease activity including adenopathy and angiotensin-converting enzyme (ACE) levels [14]. Additionally, splenic nodules may be seen in up to $15 \%$ of those with splenomegaly. Our patient's abdominal CT demonstrated splenomegaly with hypodense nodules, a rare manifestation of sarcoidosis. In our patient, the extent of neurological involvement was particularly alarming. Neurosarcoidosis occurs when granulomatous inflammation affects the central or peripheral nervous systems. Its mutable presentations include mononeuropathies, hydrocephalus, neuroendocrine disturbances, radiculopathies and peripheral nervous presentations. Sarcoidosis affects the spinal cord in $10 \%$ of those with neurosarcoidosis, with a predilection for cervical and thoracic segments. Presentations vary, since granulomatous inflammation can develop in extra- or intra-dural and intramedullary portions of the 
spine. However, paresthesias and weakness are the most common presenting symptoms [15], Neurosarcoidosis may also affect peripheral large or small nerve fibers, with large-fiber involvement presenting, as in our patient, with axonal degeneration and occasional cases of demyelination.

\section{Conclusion}

Our case represents a unique combination of several unusual sarcoidosis manifestations in a single individual. Sarcoidosis is very rarely on the differential for systemic fatigue, cachexia, splenomegaly and severe neurological degeneration. However, our case demonstrates the degree of systemic involvement that may result from this highly variable disease, and we hope that it sensitizes physicians to the protean nature of sarcoidosis extensive manifestations.

\section{References}

1. Thomas KW, Hunninghake GW (2003) Sarcoidosis. JAMA 289: 3300-3303.

2. Agostini C, Adami F, Semenzato $G(2000)$ New pathogenetic insights into the sarcoid granuloma. Curr Opin Rheumatol 12:71-76.

3. Baughman RP, Culver DA, Judson MA (2011) A concise review of pulmonary sarcoidosis. Am J Respir Crit Care Med 183:573-581.

4. Sharma OP (1999) Fatigue and sarcoidosis. Eur Respir J 13: 713.
5. Baughman RP, Teirstein AS, Judson MA, Rossman MD, Yeager $\mathrm{H} \mathrm{Jr}$, et al. (2001) Clinical characteristics of patients in a case control study of sarcoidosis. Am J Respir Crit Care Med 164: 1885-1889.

6. Valeyre D, Bernaudin JF, Uzunhan Y, Kambouchner M, Brillet PY, et al. (2014) Clinical presentation of sarcoidosis and diagnostic workup. Semin Respir Crit Care Med 35: 336-351.

7. Ramachandraiah V, Aronow W, Chandy D (2017) Pulmonary sarcoidosis: An update. Postgrad Med 129: 149-158.

8. English JC, Patel PJ, Greer KE (2001) Sarcoidosis. J Am Acad Dermatol 44 725-743.

9. Jamilloux $Y$ (2014) Sarcoidosis and uveitis. Autoimmun Rev 13: 840-849.

10. Kron J, Ellenbogen KA (2015) Cardiac sarcoidosis: Contemporary review. J Cardiovasc Electrophysiol 26: 104-109.

11. Kellner $H$ (1992) Ultrasound findings in Lofgren's syndrome: Is ankle swelling caused by arthritis,tenosynovitis, or periarthritis? J Rheumatol 19: 38.

12. Warshauer DM, Dumbleton SA, Molina PA, Yankaskas BC, Parker LA, et al. (1994) Abdominal CT findings in sarcoidosis: Radiologic and clinical correlation. Radiology 192: 93-98.

13. Joseph FG (2007) Sarcoidosis of the nervous system. Pract Neurol 7: 234-244.

14. Terushkin V, Stern BJ, Judson MA, Hagiwara M, Pramanik B, et al. (2010) Neurosarcoidosis: Presentations and management. Neurologist 16: 2-15.

15. Burns TM, Dyck PJ, Aksamit AJ, Dyck PJ (2006) The natural history and longterm outcome of 57 limb sarcoidosis neurology cases. J Neurol Sci 244: 77-87. 